\title{
A TRANSFER FOR COMPACT LIE GROUP ACTIONS
}

\author{
ROBERT OLIVER
}

\begin{abstract}
A short construction of a transfer homomorphism from $H_{*}(X / G)$ to $H_{*}(X / H)$, where $H \subseteq G$ are compact Lie groups and $X$ is a $G$-CW-complex, is given.
\end{abstract}

We give here a quick construction of a transfer homomorphism for compact Lie group actions due to Lewis, May and McClure [2]. More specifically, we sketch a shortened version of their proof of

THEOREM. Let $G$ be a compact Lie group, $H \subseteq G$ a closed subgroup, and $X$ a $G-C W$-complex. Then there exist homomorphisms (for any coefficient group $R$ )

$$
\begin{aligned}
& \operatorname{trf}: H_{*}(X / G ; R) \rightarrow H_{*}(X / H ; R) \text { and } \\
& \operatorname{trf}^{*}: H^{*}(X / H ; R) \rightarrow H^{*}(X / G ; R),
\end{aligned}
$$

natural in $X$; and such that $\pi_{*} \circ \operatorname{trf}$ and $\operatorname{trf}^{*} \circ \pi^{*}$ are multiplication by $\chi(G / H)$. Here, $\pi:(X / H) \rightarrow X / G$ denotes the projection.

For convenience, we concentrate on the homology transfer-the construction in cohomology is the same. Let $G$ be a fixed compact Lie group. By $G$-representation will always be meant a finite dimensional representation over $\mathbf{R}$. For any $G$ representation $V, S^{V}$ will denote its one-point compactification. If $X$ is a $G$ CW-complex with basepoint $x_{0} \in X^{G}$, then we write $\Sigma^{V} X=S^{V} \wedge X$ for its $V$-suspension.

The following "skeletal approximation theorem" for suspensions will be needed.

LEMMA. Let $X, Y$ be pointed $G-C W$-complexes, and let $V$ be a $G$-representation. Then any $G$-map $f: \Sigma^{V} X \rightarrow \Sigma^{V} Y$ is $G$-homotopic to a "skeletal" map $f^{\prime}$; i.e.., $f^{\prime}\left(\Sigma^{V}\left(X^{n}\right)\right) \subseteq \Sigma^{V}\left(Y^{n}\right)$ for all $n \geq 0$.

Proof. For each $n, \Sigma^{V}\left(X^{n}\right)$ is constructed by attaching, to $\Sigma^{V}\left(X^{n-1}\right)$, pairs of the form

$$
\begin{aligned}
\left(D(V) \times G / H \times D^{n},\right. & \left.\left(S(V) \times G / H \times D^{n}\right) \cup\left(D(V) \times G / H \times S^{n-1}\right)\right) \\
& \simeq\left(G / H \times D\left(V \times \mathbf{R}^{n}\right), G / H \times S\left(V \times \mathbf{R}^{n}\right)\right) .
\end{aligned}
$$

Here, $D(-)$ and $S(-)$ denote unit disk and sphere, and "attaching the pair $\left(X_{1}, X_{2}\right)$ " means attaching $X_{1}$ via a map defined on $X_{2}$. Furthermore, for any $G$-space $Z$, there is a bijection

$$
\operatorname{Map}_{G}\left(G / H \times D\left(V \times \mathbf{R}^{n}\right), Z\right) \simeq \operatorname{Map}_{H}\left(D\left(V \times \mathbf{R}^{n}\right), Z\right)
$$

(note that $G / H \times D\left(V \times \mathbf{R}^{n}\right) \simeq G \times_{H} D\left(V \times \mathbf{R}^{n}\right)$ ).

Received by the editors July 25, 1984 and, in revised form, July 9, 1985.

1980 Mathematics Subject Classification. Primary 57S10; Secondary 54E60, 55N25.

Key words and phrases. Compact Lie group action, transfer homomorphism, orbit spaces, equivariant $\mathrm{CW}$-complexes. 
It thus suffices to prove, for any $H \subseteq G$, any $n \geq 0$, any $H$-equivariant map $\varphi: D\left(V \times \mathbf{R}^{n}\right) \rightarrow S^{V} \times Y$, and any $H$-homotopy $\Psi=\left(\psi_{t}\right)$ of $\psi=\varphi \mid S\left(V \times \mathbf{R}^{n}\right)$ with $\operatorname{Im}\left(\psi_{1}\right) \subseteq S^{V} \wedge Y^{n-1}$, that $\Psi$ extends to a homotopy $\Phi=\left(\varphi_{t}\right)$ of $\varphi$ such that $\operatorname{Im}\left(\varphi_{1}\right) \subseteq S^{V} \wedge Y^{n}$. For each $K \subseteq H$, set

$$
d_{K}=\operatorname{dim}\left(V \times \mathbf{R}^{n}\right)^{K}=n+\operatorname{dim}\left(V^{K}\right) .
$$

If the pair $\left(\left(S^{V} \wedge Y\right)^{K},\left(S^{V} \wedge Y^{n}\right)^{K}\right)$ is $d_{K}$-connected for all $K \subseteq H$, then the construction of $\Phi$ is immediate. But $\left(S^{V} \wedge Y\right)^{K}$ is obtained by attaching, to $\left(S^{V} \wedge Y^{n}\right)^{K}$, pairs of the form

$$
\begin{aligned}
\left(D(V)^{K} \times\left(G / K_{i}\right)^{K} \times D^{n+j},\right. & \left(S(V)^{K} \times\left(G / K_{i}\right)^{K} \times D^{n+j}\right) \\
& \left.\cup\left(D(V)^{K} \times\left(G / K_{i}\right)^{K} \times S^{n+j-1}\right)\right) \\
\cong & \left(G / K_{i}\right)^{K} \times\left(D^{d_{K}+j}, S^{d_{K}+j-1}\right)
\end{aligned}
$$

for $K_{i} \subseteq G$ and $j \geq 1$, so $\left(\left(S^{V} \wedge Y\right)^{K},\left(S^{V} \wedge Y^{n}\right)^{K}\right)$ is $d_{K}$-connected.

The main step of the construction is the following "homology desuspension" result.

Proposition. Let $V$ be a G-representation, $X$ and $Y$ (pointed) G-CWcomplexes, and $f: \Sigma^{V} X \rightarrow \Sigma^{V} Y$ a $G$-map. Then there is an induced homomorphism (for any coefficient group $R$ ) $f_{\#}: \tilde{H}_{*}(X / G ; R) \rightarrow \tilde{H}_{*}(Y / G ; R)$, which is natural in $f$, and has the following properties:

(1) $\left(\Sigma^{W} f\right)_{\#}=f_{\#}$ for any $G$-representation $W$.

(2) $f_{\#}=(f / G)_{*}$ if $f: X \rightarrow Y$ (i.e., $\left.V=0\right)$.

(3) If $\varphi: S^{V} \rightarrow S^{V}$ is a $G$-map of (ordinary) degree $k$, then $\left(\varphi \wedge \operatorname{id}_{X}\right)_{\#}$ is multiplication by $k$.

PROOF. We may assume (suspending if necessary) that $G$ acts orientably on $V$. Fix a map $f: \Sigma^{V} X \rightarrow \Sigma^{V} Y$. By the lemma, $f$ is homotopic to a map $f^{\prime}$ such that $f^{\prime}\left(\Sigma^{V}\left(X^{n}\right)\right) \subseteq \Sigma^{V}\left(Y^{n}\right)$ for all $n$.

Let $k=\operatorname{dim}(V)$. If

$$
X^{n}=X^{n-1} \cup_{\varphi}\left(\coprod_{i} G / H_{i} \times D^{n}\right) \quad \text { and } \quad Y^{n}=Y^{n-1} \cup_{\psi}\left(\coprod_{j} G / K_{j} \times D^{n}\right)
$$

(and $X^{-1}, Y^{-1}$ are the basepoints), then

$$
\left.H_{n+k}\left(\Sigma^{V}\left(X^{n}\right)\right), \Sigma^{V}\left(X^{n-1}\right) ; R\right) \cong H_{n}\left(X^{n}, X^{n-1} ; R\right) \cong \sum_{i} H_{0}\left(G / H_{i} ; R\right)
$$

and

$$
H_{n+k}\left(\Sigma^{V}\left(Y^{n}\right), \Sigma^{V}\left(Y^{n-1}\right) ; R\right) \cong H_{n}\left(Y^{n}, Y^{n-1} ; R\right) \cong \sum_{j} H_{0}\left(G / K_{j} ; R\right) .
$$

Hence, dividing out linearly with the action of $\bar{G}=\pi_{0}(G)$, we can identify

$$
\begin{aligned}
R \otimes_{R[\bar{G}]} H_{n+k}\left(\Sigma^{V}\left(X^{n}\right), \Sigma^{V}\left(X^{n-1}\right)\right) & \cong \sum_{i} R \cong H_{n}\left(X^{n} / G, X^{n-1} / G ; R\right) \\
& =C_{n}(X / G ; R)
\end{aligned}
$$


and similarly for $Y$. So $f^{\prime}$ induces homomorphisms (for all $n \geq 0$ )

$$
\hat{f}_{n}^{\prime}=R \otimes_{R[\bar{G}]} H_{n+k}\left(f_{n}^{\prime}, f_{n-1}^{\prime}\right): C_{n}(X / G ; R) \rightarrow C_{n}(Y / G ; R) .
$$

These clearly commute with the boundary maps and hence induce

$$
f_{\#}=f_{\#}^{\prime}: \tilde{H}_{n}(X / G ; R) \rightarrow \tilde{H}_{n}(Y / G ; R)
$$

If $f^{\prime \prime}$ is another skeletal approximation to $f$, then let $F$ be a skeleton preserving homotopy from $f^{\prime}$ to $f^{\prime \prime}$. Then $\hat{F}_{n}$ (in the above notation) defines a chain homotopy between $\hat{f}_{n}^{\prime}$ and $\hat{f}_{n}^{\prime \prime}$. It follows that $f_{\#}$ is uniquely defined independently of the choice of $f^{\prime}$.

Naturality of $f_{\#}$ and conditions (1) and (2) are now clear. If $\varphi: S^{V} \rightarrow S^{V}$ is any $G$-map, then

$$
\left(\varphi \wedge \operatorname{id}_{X}\right)_{*}: H_{n+k}\left(\Sigma^{V}\left(X^{n}\right), \Sigma^{V}\left(X^{n-1}\right)\right) \rightarrow H_{n+k}\left(\Sigma^{V}\left(X^{n}\right), \Sigma^{V}\left(X^{n-1}\right)\right)
$$

is multiplication by $\operatorname{deg}(\varphi)$ for any $n \geq 0$; and hence $\left(\varphi \wedge \operatorname{id}_{X}\right)_{\#}$ is multiplication by $\operatorname{deg}(\varphi)$.

The construction of the transfer now follows easily. Fix $H \subseteq G$, and let $V$ be any $G$-representation having an embedding $G / H \subseteq V$. Let $t$ denote the composite

$$
t: S^{V} \rightarrow T\left(\nu_{G / H}\right) \subseteq T\left(\nu_{G / H} \oplus \tau_{G / H}\right)=G / H^{+} \wedge S^{V},
$$

where $T\left(\nu_{G / H}\right)$ is the Thom space of the normal bundle, and the first map the Pontrjagin-Thom construction. Note that the composite

$$
S^{V} \stackrel{t}{\rightarrow} G / H^{+} \wedge S^{V} \stackrel{\text { proj }}{\rightarrow} S^{V}
$$

has degree $\chi(G / H)$ (see [1, Theorem 2.4]).

If $X$ is any (nonbased) $G$-CW-complex, set

$$
t_{X}=t \wedge \operatorname{id}_{X}: \Sigma^{V}\left(X^{+}\right) \rightarrow G / H^{+} \wedge S^{V} \wedge X^{+} \cong \Sigma^{V}(G / H \times X)^{+} .
$$

Since $G / H \times X$ has the $G$-homotopy type of a $G$-CW-complex (Lemma 1 ) and $(G / H \times X) / G \cong X / H$, we can now define the transfer for $X$ by

$$
\operatorname{trf}=\left(t_{X}\right)_{\#}: H_{*}(X / G ; R) \rightarrow H_{*}((G / H \times X) / G ; R)=H_{*}(X / H ; R) .
$$

Naturality of trf follows from the naturality of the homology desuspension. That $\pi_{*} \circ$ trf is multiplication by $\chi(G / H)$ follows from (3) in the proposition.

\section{REFERENCES}

1. J. Becker and D. Gottlieb, The transfer map and fiber bundles, Topology 14 (1975), 1-12.

2. G. Lewis, J. P. May and J. McClure, Ordinary $R O(G)$-graded cohomology, Bull. Amer. Math. Soc. (N.S.) 4 (1981), 208-212.

Mathematisk Institut, Ny MUnkegade, Aarhus Universitet, 8000 Aarhus C, DENMARK 